\title{
Ligand Migration in the Gaseous Insulin-CB7 Complex-A Cautionary Tale About the Use of ECD-MS for Ligand Binding Site Determination
}

\author{
Brittany L. Heath, Rebecca A. Jockusch \\ Department of Chemistry, University of Toronto, Toronto, Ontario, M5S 3H6, Canada
}

\begin{abstract}
Knowledge of the structure of protein-ligand complexes can aid in understanding their roles within complex biological processes. Here we use electrospray ionization (ESI) coupled to a Fourier transform ion cyclotron resonance mass spectrometer to investigate the noncovalent binding of the macrocycle cucurbit[7]uril (CB7) to bovine insulin. Recent condensed-phase experiments (Chinai et al., J. Am. Chem. Soc. 133:8810-8813, 2011) indicate that CB7 binds selectively to the $\mathrm{N}$-terminal phenylalanine of the insulin B-chain. Competition experiments employing ESI mass spectrometry to assess complex formation between CB7 and wild type insulin B-chain vs. a mutant B-chain, confirm that the $\mathrm{N}$-terminal phenylalanine plays in important role in solution-phase binding. However, analysis of fragment ions produced by electron capture dissociation (ECD) of CB7 complexed to intact insulin and to the insulin B-chain suggests a different picture. The apparent gas-phase binding site, as identified by the ECD, lies further along the insulin B-chain. Together, these studies thus indicate that the CB7 ligand migrates in the ESI mass spectrometry analysis. Migration is likely aided by the presence of additional interactions between CB7 and the insulin B-chain, which are not observed in the crystal structure. While this conformational difference may result simply from the removal of solvent and addition of excess protons by the ESI, we propose that the migration may be enhanced by charge reduction during the ECD process itself because ion-dipole interactions are key to CB7 binding. The results of this study caution against using ECD-MS as a stand-alone structural probe for the determination of solution-phase binding sites.
\end{abstract}

Key words: Electron capture dissociation, Fourier-transform ion cyclotron resonance mass spectrometry, Ligand binding site determination, Insulin, Cucurbituril, Ion activation

\section{Introduction}

$\mathrm{P}$ roteins play a vital role in a vast number of important biological processes. The function that an individual protein has is defined not only by the structure of the

Electronic supplementary material The online version of this article (doi:10.1007/s13361-012-0470-3) contains supplementary material, which is available to authorized users.

Correspondence to: Rebecca A. Jockusch; e-mail: rebecca.jockusch@ utoronto.ca protein, but by its interactions and associations with other proteins, macromolecules, and/or ligands. Such noncovalent complexes are stabilized by hydrogen bonds, electrostatic interactions, and/or van der Waals interactions as well as by the hydrophobic effect. Analysis of these interactions and knowledge of the structure of the complex is often critical in understanding biological processes and can significantly aid in the establishment of new drug targets.

Mass spectrometry coupled with electrospray ionization (ESI-MS) has become a well-established technique for the study of noncovalent complexes of proteins [1-3]. With careful control of experimental conditions, noncovalent 
protein-ligand $[4,5]$ or protein-protein complexes [2, 6-9] can be transferred intact into the gas phase. Subsequent molecular mass measurements provide direct evidence of the presence of the complex, as well as the stoichiometry of the binding. Additional information about proteins and their complexes, including protein sequence information, can be obtained from tandem mass spectrometry (MS/MS) experiments. Collisionally activated dissociation (CAD) is the central technology for MS/MS; however, many noncovalent complexes and even many covalent protein modifications usually dissociate when subjected to collisions, rendering CAD inadequate for tasks such as the determination of ligand binding sites. To circumvent this problem, MS coupled with chemical crosslinking $[10,11]$ or footprinting approaches $[12,13]$ have been used to further elucidate direct interactions and overall topology of multiprotein complexes using CAD methods. However, these approaches are often time-consuming and are not applicable to all protein systems. For example, with chemical crosslinking, a protein can go unnoticed by the chemical linker if it does not have the right functional group.

The development of electron capture dissociation (ECD) [14] was a key advance in tandem MS. In ECD, and the closely related technique known as electron transfer dissociation (ETD) [15], the capture of a low energy electron by the analyte of interest is used to effect rapid fragmentation. Not only does ECD provide greater sequence coverage than CAD methods, it has been successful in localizing covalent protein modifications [16-19]. Moreover, ECD has been shown in many cases to preferentially cleave the protein backbone without disruption of noncovalent interactions [20, 21]. This technique has been successfully applied in the localization of binding sites for several noncovalent complexes [22-27]. In each of these studies, the gas-phase results have matched closely with previous solution-phase studies on the complexes, thus the use of ECD for structural determination of non-covalent complexes has shown great promise. For example, in a groundbreaking paper published by Loo et al. [22] in 2006, the authors were able to localize the binding site of spermine, a small amine shown to promote aggregation of $\alpha$-synuclein, to the $\mathrm{C}$-terminal region of $\alpha$-synuclein, which was consistent with NMR studies. It is important to remember, however, that because the gas-phase ions are completely free of solvent, the ensemble of conformations that are adopted by a protein in a mass spectrometer may differ from that of the same protein in solution. As a result, differences in the structure and energetics of protein-ligand interactions between solutionphase and gas-phase studies are possible.

A recent study on the heme-binding properties of myoglobin using ETD-MS presents results that are somewhat different than the solution-phase picture [28]. Depending on the charge state of precursor holo-myoglobin, Enyenihi et al. [28] observed differences in the apparent preferred binding interaction of the heme. ETD of high charge state $(16+)$ heme-myoglobin complex produced fragments that indicated preferential binding of the heme to His93, which is the strongest binding (proximal) ligand in the condensed-phase binding site. However, upon ETD of a lower charge state $(12+)$, which should have a structure more closely resembling that in solution than the higher charge state, the heme was found to preferentially remain bound to His64, which is the weaker (distal) solution-phase ligand. These differences were attributed to the lack of water and multiple protonation sites in the gas phase. Despite these unanticipated findings, the overall results of the ECD-MS study by Enyenihi et al. were broadly consistent with condensed-phase structure of myoglobin in that the heme was found to be coordinated by the two expected histidine residues.

Here, we use ECD to examine the complex between insulin and the synthetic receptor cucurbit[7]uril (CB7) (Figure 1). Using isothermal titration calorimetry (ITC) and fluorescence spectroscopy, Chinai et al. [29] demonstrated that CB7 binds specifically $\left(\mathrm{K}_{\mathrm{a}}=1.5 \times 10^{6} \mathrm{M}^{-1}\right)$ and selectively to the $\mathrm{N}$ terminal phenylalanine (Phe) of the insulin B-chain. Convincing evidence for the specificity of $\mathrm{CB} 7$ for the $\mathrm{N}$-terminal Phe residue was provided by demonstrating a $>1000$-fold binding selectivity of native insulin over a mutant insulin, which contained a glutamic acid in place of Phe at the $\mathrm{N}$-terminus of the B-chain. The selectivity of binding, which had been previously demonstrated for small peptides containing $\mathrm{N}$ terminal aromatic residues (i.e., Phe, Tyr, or Trp) [30], is attributed to the fact that an N-terminal aromatic residue fixes the aromatic and ammonium group in close proximity. This provides favorable ion-dipole interactions between the portal oxygen atoms of the $\mathrm{CB} 7$ macrocycle and the charge on the ammonium group, combined with favorable van der Waals interactions between the nonpolar interior of CB7 and the aromatic side chain upon formation of an inclusion complex. An X-ray crystal structure of the complex shows that the $\mathrm{N}$-terminus unfolds from the surface of the protein to accommodate the binding of the macrocyclic host molecule (Figure 1c) [29].

Here, we employ ESI-MS in combination with ECD for analysis of the binding site of $\mathrm{CB} 7$ to bovine insulin. Contrary to our expectations, we found that the transfer of this complex from solution to the gas phase and subsequent ECD proceeds with some significant structural changes, and that the apparent binding site, as determined by ECD-MS experiments, is different from the accepted condensed-phase binding site.

\section{Experimental}

\section{Materials}

Insulin from bovine pancreas (product number I5500, neutral experimental monoisotopic mass, $\mathrm{M}_{\mathrm{m} \text {,expt }}=5729.63$, $\left.\mathrm{M}_{\mathrm{m} \text {,theor }}=5729.60 \mathrm{Da}\right)$ and cucurbit[7]uril $\left(\mathrm{M}_{\mathrm{m} \text {,expt }}=1162.36\right.$, $\left.\mathrm{M}_{\mathrm{m} \text {,theor }}=1162.34 \mathrm{Da}\right)$ were purchased from Sigma-Aldrich (St. Louis, MO, USA). Stock solutions of insulin were prepared 
(a)

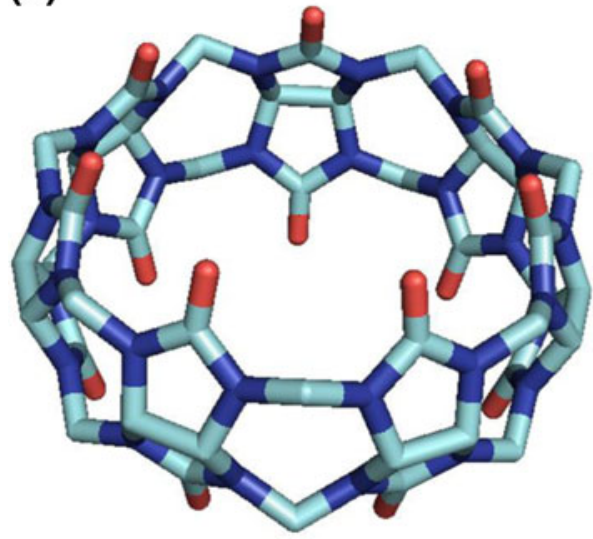

(b)

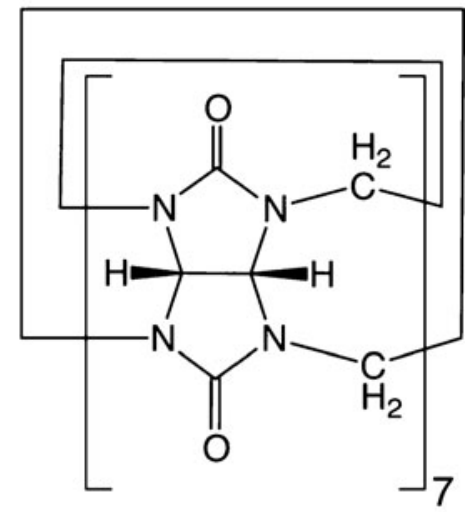

(c)

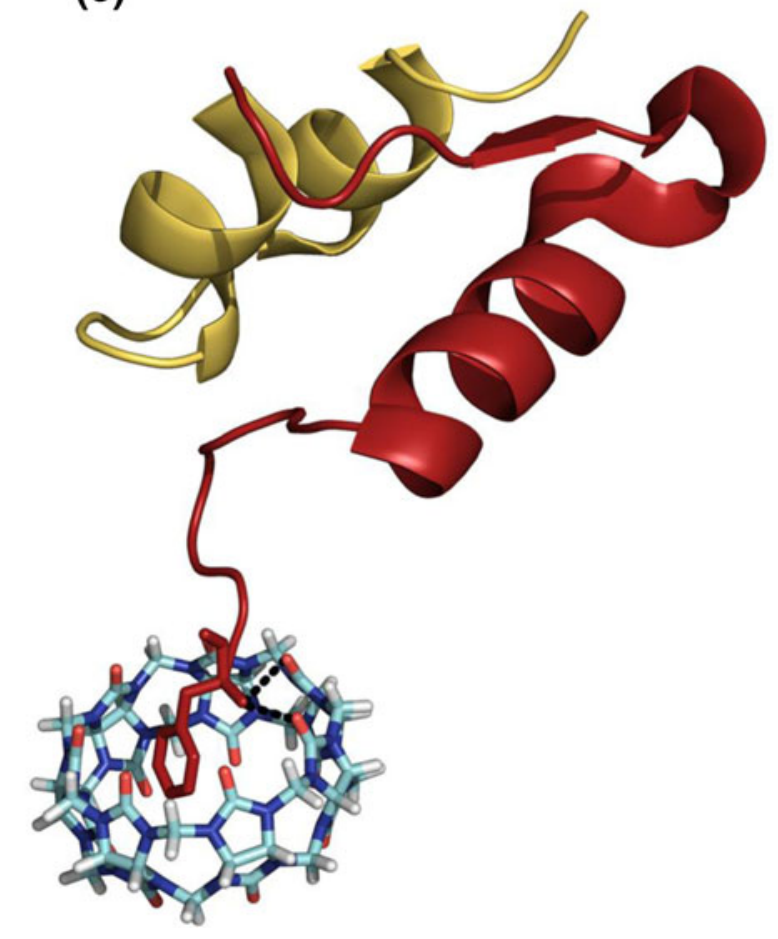

Figure 1. (a) Structure of cucurbit[7]uril. Oxygen atoms are shown in red, carbon atoms in light blue, and nitrogen atoms in dark blue. Hydrogen atoms have been omitted from the structure for clarity. (b) Chemical scheme of cucurbituril showing the glycoluril unit. (c) Crystal structure of insulin bound to CB7 (Protein Data Bank Structure: 3Q6E from ref. 29), showing the binding of $\mathrm{CB} 7$ to the $\mathrm{N}$-terminal phenylalanine on the B-chain. The B-chain is shown in red, and the A-chain in yellow. (Image rendered using PyMOL [43])

by dissolving the lyophilized powder in $\mathrm{pH} 2 \mathrm{HCl}$ to a concentration of $500 \mu \mathrm{M}$. Dilute solutions were prepared in $10 \mathrm{mM}$ ammonium acetate, adjusted to $\mathrm{pH} 7.4$ with ammonium hydroxide; $500 \mu \mathrm{M}$ stock solutions of $\mathrm{CB} 7$ in water were sonicated for 5 minutes to ensure dissolution; CB7 was added to dilute solutions of bovine insulin to produce a final concentration of $50 \mu \mathrm{M}$ CB7:25 $\mu \mathrm{M}$ insulin unless otherwise indicated.

Reduced insulin B-chain was obtained by reduction using dithiothreitol (DTT), obtained from Sigma-Aldrich. A solution of $40 \mu \mathrm{M}$ insulin and $10 \mathrm{mM}$ DTT was heated to $70{ }^{\circ} \mathrm{C}$ with stirring for 10 minutes. ESI mass spectra confirmed the complete reduction of all three disulfide bonds and the presence of both the insulin $\mathrm{A}$ and B chain (reduced B-chain $\mathrm{M}_{\mathrm{m} \text {,expt }}=3397.68$, $\left.\mathrm{M}_{\mathrm{m} \text {,theor }}=3397.67 \mathrm{Da}\right)$.

Mutant insulin B-chain, in which the N-terminal Phe is replaced by glycine (Gly) (Phe ${ }^{\mathrm{B} 1} \mathrm{Gly}$ ), was obtained from BioMatik (Cambridge, ON, Canada) as a lyophilized powder. A $500 \mu \mathrm{M}$ stock solution of the mutant B-chain was prepared in $10 \mathrm{mM}$ ammonium acetate adjusted to $\mathrm{pH}$ 7.4 with ammonium hydroxide. Disulfide bond reduction was carried out using the procedure described above for bovine insulin $\left(\mathrm{M}_{\mathrm{m} \text {,expt }}=3307.64, \mathrm{M}_{\mathrm{m} \text {,theor }}=3307.62 \mathrm{Da}\right)$. 


\section{Mass Spectrometry}

Mass spectrometry experiments were performed using a $7 \mathrm{~T}$ Apex Qe Fourier transform ion cyclotron resonance (FTICR) mass spectrometer (Bruker Daltonics, Billerica, MA, USA) equipped with an Apollo II source set up for nanoelectrospray ionization (nano-ESI). The nano-ESI emitter consisted of a borosilicate glass capillary (1.0 mm o.d., $0.78 \mathrm{~mm}$ i.d.), which had been pulled to a 5-10 $\mu \mathrm{m}$ opening at one end using a micropipette puller (model P97; Sutter Instruments, Novato, CA, USA). A platinum wire inserted into the glass tip was held at ground, while a voltage ranging from $-900 \mathrm{~V}$ to $-1100 \mathrm{~V}$ was applied to the entrance of the mass spectrometer to generate the positive mode electrospray. The source and ion transfer voltages were adjusted to enhance ion signal for the CB7 complex and were kept constant for all experiments. Many noncovalent complexes, including insulin hexamers, have been detected under the same source conditions on our instrument, so we believe that the conditions used were relatively gentle.

Desolvated ions passed through a quadrupole and were accumulated in the "collision" hexapole (h2) for $0.8-1.0 \mathrm{~s}$. This hexapole is operated at an elevated pressure $\left(\sim 10^{-3}\right.$ mbar) of Argon. Unless otherwise noted, the collision voltage was maintained at $0 \mathrm{~V}$ (i.e., no potential bias was applied to effect energetic collisions within h2). After the accumulation period, ions were transmitted to the ICR cell for subsequent tandem mass spectrometry experiments.

Electron capture dissociation experiments were carried out in the ICR cell on charge-isolated species. The isolation was performed first in the mass selective quadrupole (which is located before the accumulation cell) with a $\mathrm{m} / \mathrm{z}$ window ranging from 5 to $20 \mathrm{~m} / \mathrm{z}$ depending on the ion intensity. The ions were then further isolated after transfer to the ICR cell, with an isolation power of ranging from $15 \%$ to $17 \%$ and a safety belt parameter of $1 \mathrm{~m} / \mathrm{z}$. Ions stored in the ICR cell were subjected to low energy electrons by applying a current of 1.3 A through a hollow cathode heater. The electrons were emitted with a pulse duration of $80 \mathrm{~ms}$. A $1.0 \mathrm{~V}$ electron beam bias and a $15 \mathrm{~V}$ lens potential were used to guide and focus the electrons into the ICR cell. Each ECD spectrum was the sum of 200, $0.7078 \mathrm{~s}$ transients, each with $512 \mathrm{~K}$ data points. The mass spectra were externally calibrated using ES tuning mix (Agilent Tech., Mississauga, ON, Canada) and analyzed using DataAnalysis and IsotopePattern software (Bruker Daltonics, Billerica, MA, USA) The SNAP 2.0 algorithm was used for automated peak picking, and the resulting fragment mass lists were searched against the primary sequence of each peptide using the Prosight-PTM software package [31].

\section{Results and Discussion}

\section{Nano-ESI-MS of Insulin and CB7}

Figure 2 shows a typical nano-ESI mass spectrum of a solution containing $25 \mu \mathrm{M}$ bovine insulin and $50 \mu \mathrm{M}$ CB7. Strong signal from the 1:1 complex (holo insulin), in both the $4+$ and $5+$ charge states, is observed. In addition, $\sim 10 \%$ of the ion intensity corresponds to insulin without CB7 bound (apo-insulin). There is no evidence of a 1:2 complex. This agrees well with the condensed-phase results [29], indicative of the presence of a single high affinity binding site for CB7 on insulin. Based on the $0.66 \mu \mathrm{M} \mathrm{K}_{\mathrm{d}}$ determined by Chinai et al., insulin should exist predominantly $(\sim 99 \%)$ as the complex in solution at the concentrations used here. It is evident that this is not directly translated to the gas phase from the ESI mass spectrum shown in Figure 2, which shows that only $290 \%$ of insulin exists in complex with CB7. This could be a result of using ammonium acetate buffer in our studies in place of sodium phosphate buffer, given that ammonium ions have a strong affinity for CB7 [32, 33], or it could suggest that some complexes are falling apart in the ESI mass spectrometry process. At higher concentrations of CB7 ligand $(100 \mu \mathrm{M})$ and bovine insulin $(50 \mu \mathrm{M})$ there was a small amount ( $\sim 5 \%)$ of signal intensity from the 1:2 complex (data not shown), which could be a result of non-specific binding at higher concentrations.

\section{Competitive Binding Experiment}

Figure 3 shows the results of a competitive binding experiment between reduced wild-type insulin B-chain $\left(\mathrm{B}_{\text {red }}\right)$ and a mutant B-chain in which the N-terminal Phe is replaced by Gly. Several N-terminal mutations, including the Phe ${ }^{\mathrm{B} 1}$ Gly mutation, appear to have only a minor effect of the conformation of the B-chain [34]. This may be attributable to the flexibility of the $\mathrm{N}$-terminal region of the B-chain observed in nuclear magnetic resonance (NMR) experiments under a range of solution conditions [35]. Figure 3a shows the nano-ESI mass spectrum of equimolar concentrations $(10 \mu \mathrm{M})$ of reduced wild-type and mutant B-chain in the absence of CB7. The intensities of the two peptides are similar, indicating minimal differences in ionization efficiencies. Figure 3b-d) show the effect of increasing the concentration of $\mathrm{CB} 7$ from 10 to $50 \mu \mathrm{M}$, while keeping the concentration of each peptide constant at $10 \mu \mathrm{M}$. An increase in the intensity of the complex containing the reduced wild-type B-chain is clearly observed, accompanied by a subsequent decrease in the intensity of the unbound $\mathrm{B}_{\text {red }}$ signal. Over this concentration range, no CB7 complex with the mutant B-chain peptide is observed in either the competitive binding experiments (Figure 3), or in noncompetitive analyses (data not shown). These results clearly indicate that in solution, the N-terminal phenylalanine of the insulin B-chain is required for high affinity interaction with CB7.

An increase in most favored charge state, from $4+$ to $5+$, is observed upon CB7 binding. This is true for the both reduced B-chain (Figure 3) and for intact insulin (see Figure 2). The increase in charge state may simply be due to the increased size of the complex; however, it may suggest that the presence of CB7 helps to stabilize 


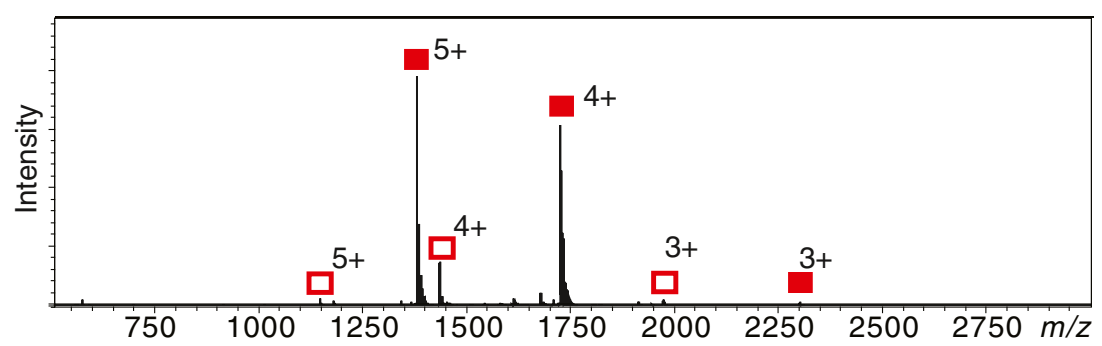

Figure 2. Nano-ESI mass spectrum of $25 \mu \mathrm{M}$ bovine insulin and $50 \mu \mathrm{M}$ CB7 sprayed out of $10 \mathrm{mM}$ ammonium acetate, adjusted to $\mathrm{pH}$ 7.4. Open squares represent unbound (apo) insulin, and the closed circles represent the 1:1 complex (holo insulin). No evidence of any 1:2 complex is observed

(provide solvation for) multiple charges in the gas-phase conformation.

\section{Electron Capture Dissociation of Insulin-CB7 Complexes}

Figure 4a illustrates the results of ECD of the [Ins + CB7 + $5 \mathrm{H}]^{5+}$ complex (see also Figure S1). Due to the low probability of breaking both interchain disulfide bonds, all assigned fragments are confined to the exocyclic regions of the protein, and there is a lack of sequence coverage within the endocyclic region. Fragments corresponding to 17 of the possible 23 exocyclic cleavage sites are observed. In the figure, a bar over the fragment name is used to indicate the fragments that have retained CB7 after ECD. Of the 24 assigned fragments, 17 of them have retained CB7. As expected, all identified fragments that result from fragmentation of the A-chain (and thus contain a portion of the Achain and the entire B-chain) retain CB7. Unexpectedly, however, six holo (with CB7) B-chain $z$-fragments are observed (z29 to z24), which suggests that the CB7 is not bound at the N-terminus, but instead somewhere else on the B-chain. Moreover, the detection of complementary apo (without CB7) c5 and c6 B-chain fragments are consistent with the analysis.

The data illustrated in Figure 4a suggest that the binding site of CB7 lies within the span of 16 residues between Cys7 and $\operatorname{Arg} 22$ on the B-chain. Unfortunately, more specific localization of the CB7 binding site on intact insulin was not possible due to the lack of sequence coverage within the endocyclic region. We are aware that Zubarev et al. [36] have previously demonstrated that the capture of a single electron can result in multiple cleavages of cyclic disulfide proteins and peptides (i.e., either an $\mathrm{S}-\mathrm{S}$ bond and a backbone bond on the same ring, or two disulfide bonds holding two peptide chains together), however, this was not observed for ECD of the complex with $\mathrm{CB} 7$ under the experimental conditions used here. The apparent CB7 binding site between Cys7 and Arg22 suggested by ECD analysis, together with the results from the competition experiment between $\mathrm{B}_{\text {red }}$ and the mutant B-chain, which are consistent with binding at the N-terminal Phe in solution, suggests that the CB7 ligand has migrated in the gas phase, either as a result of the ESI process, the accumulation and transfer of the ions within the mass spectrometer, or as a result of the ECD process itself.

To localize the gas-phase binding site more precisely, the binding of CB7 to the reduced B-chain was investigated. This experiment eliminates the complications imparted by the presence of the interchain disulfide bonds. Similar to the results from intact insulin, the nano-ESI spectrum of a solution of $25 \mu \mathrm{M}$ reduced insulin and

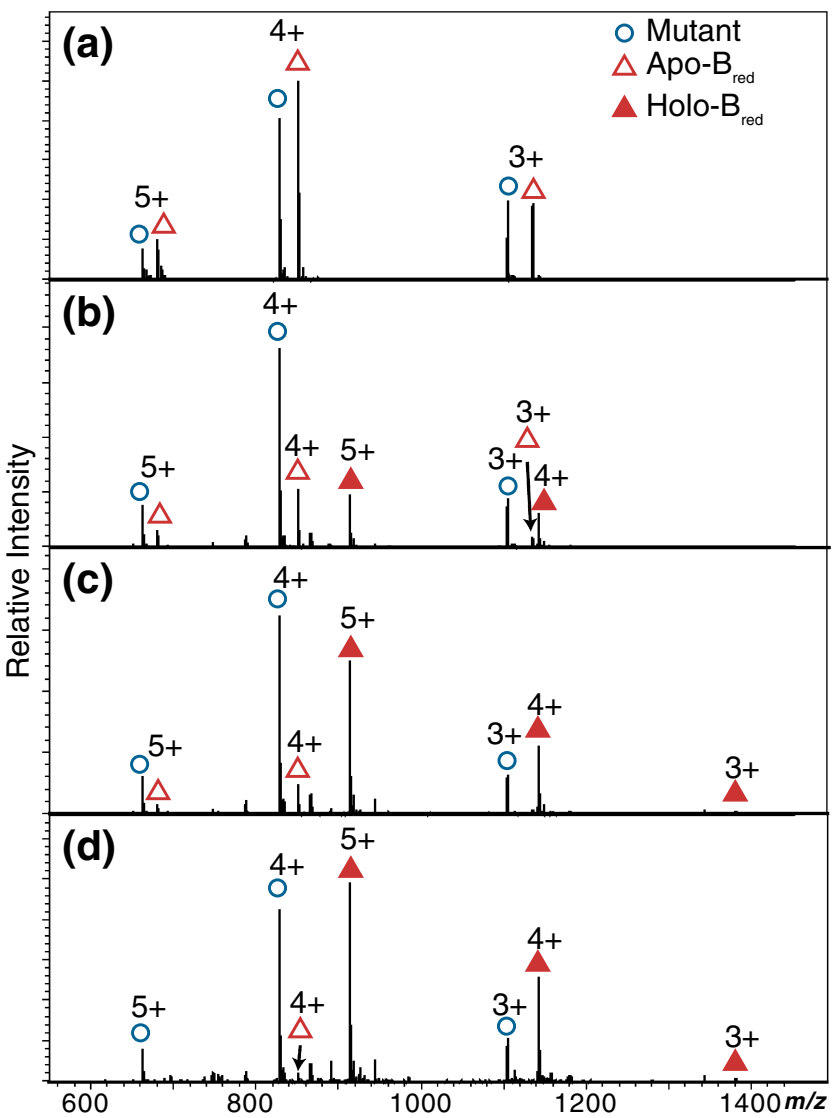

Figure 3. ESI mass spectra from competitive binding experiments between $10 \mu \mathrm{M}$ each of $B_{\text {red }}$ and Phe ${ }^{B 1}$ Gly mutant B-chain with (a) $0 \mu \mathrm{M} \mathrm{CB}$, (b) $10 \mu \mathrm{M}$ CB7, (c) $20 \mu \mathrm{M}$ CB7, and (d) $50 \mu \mathrm{M}$ CB7. The blue circles represent the mutant peptide, while the open and filled red triangles indicate apo- $\mathrm{B}_{\text {red }}$ and holo- $\mathrm{B}_{\text {red }}$, respectively 
B. L. Heath and R. A. Jockusch: Ligand Migration in the Gas Phase
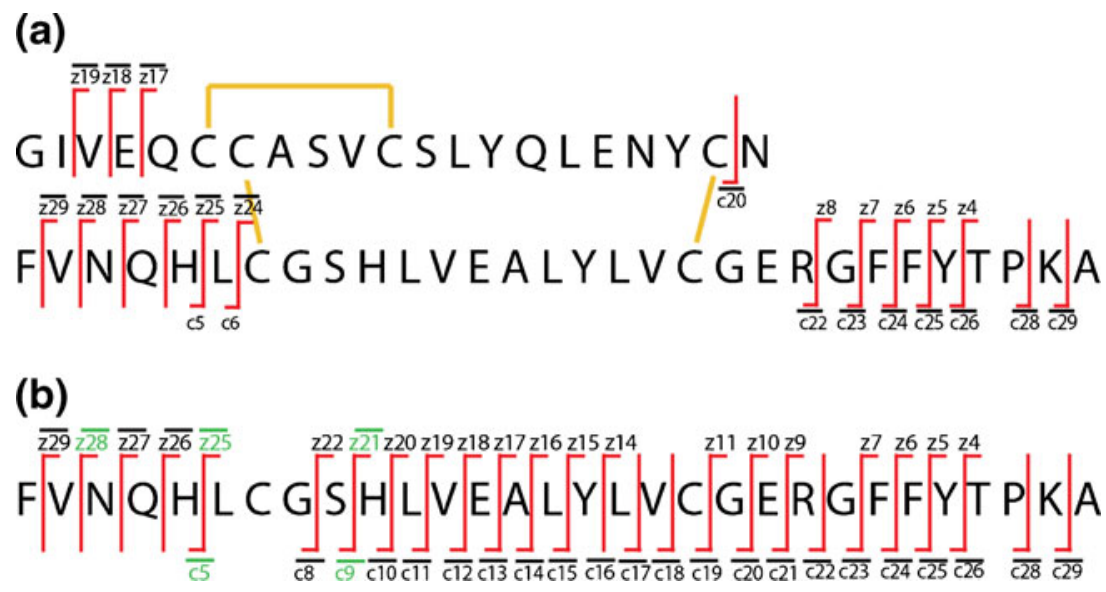

Figure 4. $E C D$ fragmentation map for (a) $[\mathrm{Ins}+\mathrm{CB} 7+5 \mathrm{H}]^{5+}$ and (b) $\left[\mathrm{B}_{\text {red }}+\mathrm{CB} 7+5 \mathrm{H}\right]^{5+}$. Disulfide bonds between the $\mathrm{A}-\mathrm{chain}$ and B-chain and within the A-chain are shown as yellow lines. The fragments that contained CB7 are represented with a bar above the fragment label. Fragments labeled in green in panel (b) were observed in both the holo and apo form

$40 \mu \mathrm{M}$ CB7 shows predominant binding of $\mathrm{CB} 7$ in a $1: 1$ ratio with $B_{\text {red }}$, with no evidence of a 1:2 complex (Figure S2). No effort was made to remove the A-chain from the solution, and as expected, there is no evidence of $\mathrm{CB} 7$ binding to the A-chain under the investigated solution conditions. Similar conclusions can also be made from the data shown in Figure 3.

Electron capture of mass-selected $\left[\mathrm{B}_{\text {red }}+\mathrm{CB} 7+5 \mathrm{H}\right]^{5+}$ results predominantly in the charge-reduced species $\left[\mathrm{B}_{\text {red }}+\right.$ $\mathrm{CB} 7+5 \mathrm{H}]^{4+\bullet}$ and $\left[\mathrm{B}_{\text {red }}+\mathrm{CB} 7+5 \mathrm{H}\right]^{3+}$, accompanied by dissociation of 27 out of $30 \mathrm{~N}-\mathrm{C}_{\alpha}$ bonds of the peptide (Figure S3). Of the $43 \mathrm{c} / \mathrm{z}$ fragments that were assigned, 28 contain the CB7 ligand (Table S1). Mapping of these fragments onto the sequence of the B-chain (Figure 4b) reveals results similar to those obtained from intact insulin; the CB7 is not retained at the N-terminus of the B-chain. In particular, the presence of fragment ions z29-z25 complexed with CB7, and the fragments z20-z14, which are never observed to complex with $\mathrm{CB} 7$, suggest localization of the CB7 not on the N-terminal Phe, but rather further down the B-chain. The reproducibility of the relative intensity of all identified fragments is illustrated in Figure 5a. Interestingly, five fragments were observed in both the holo and apo form $(z 28, z 25, z 21, c 5$, and c9), shown in Figure $4 b$ in green. The existence of these five fragments, which appear both in complex with CB7 and without, suggests either the presence of more than one stable conformation of the complex in the gas phase, or that in the conformation(s) present, CB7 interacts with multiple sites on the peptide and that multiple dissociation channels are readily accessible.

A major difference between the condensed-phase complex and the gas-phase complex produced by ESI is likely the extent of charging. There are four basic residues in the sequence of $\mathrm{B}_{\text {red }}$, allowing for a straightforward assignment of the five charges on gas-phase $\left[\mathrm{B}_{\text {red }}+\mathrm{CB} 7+5 \mathrm{H}\right]^{5+}$ to the N-terminus, His5, His10, Arg22, and Lys29. With an approximate $\mathrm{pK}_{\mathrm{a}}$ of 6.0 , the imidazole side chain of histidine is expected to be predominantly uncharged in solution at $\mathrm{pH}$
7.4. Thus, the highly-charged gas-phase structure may provide new interactions that make it favorable for $\mathrm{CB} 7$ to bind elsewhere on the peptide. Moreover, as CB7 has two carbonyl-lined portals and the N-terminal region of the Bchain is believed to be rather flexible [35], it is quite possible that the CB7 is sandwiched between two charged sites on the highly charged insulin, such as the protonated N-terminus and a protonated histidine. Unfortunately, we were unable to isolate a sufficient intensity of the $3+$ charge state of the reduced insulin B-chain (in which the histidine residues would presumably be neutral) for ECD analysis.

Returning to an analysis of each of the five fragments that were observed as both the apo and holo form, consideration of the most intense of either form suggests localization of the binding site of $\mathrm{CB} 7$ in the region between residues His5 and His 10 (Figure 5a). Within this region, there are no side chains that have demonstrated a significant affinity for $\mathrm{CB} 7$ in solution $[37,38]$. However, as mentioned above, it seems plausible that the presence of a positive charge on the imidazole side chain of His 5 and/or His 10 could provide favorable ion-dipole interactions with the portal oxygens on the CB7 in the gas phase.

The charge states of $\mathrm{c}$ and $\mathrm{z}$ fragments formed upon ECD of $\left[\mathrm{B}_{\text {red }}+\mathrm{CB} 7+5 \mathrm{H}\right]^{5+}$ are shown in Figure 6. Overall, the observed fragment ion charge states are consistent with positive charges located at both Arg22 and Lys29 and a further two positive charges located at two of the three remaining basic sites, the N-terminus, His5, or His 10 , in the charge-reduced peptide. This analysis is based primarily on the existence of a span of holo c-fragments from c10 to c20, which are observed only as $2+$ ions, while their complementary apo z-fragments (z20-z10) are also observed as $2+$ ions. Moreover, $\mathrm{z} 7-\mathrm{z} 4$ fragment ions carry a single positive charge. Strikingly, for both the c5 and c9 fragments, which appear as both the holo (consistent with Phel binding) and apo form (suggesting binding further down the chain), the holo fragment appears exclusively as the $2+$ charge state, whereas the apo fragment appears exclusively as a $1+$ charge state. In addition, the apo z 25 and z 21 fragments, which are 

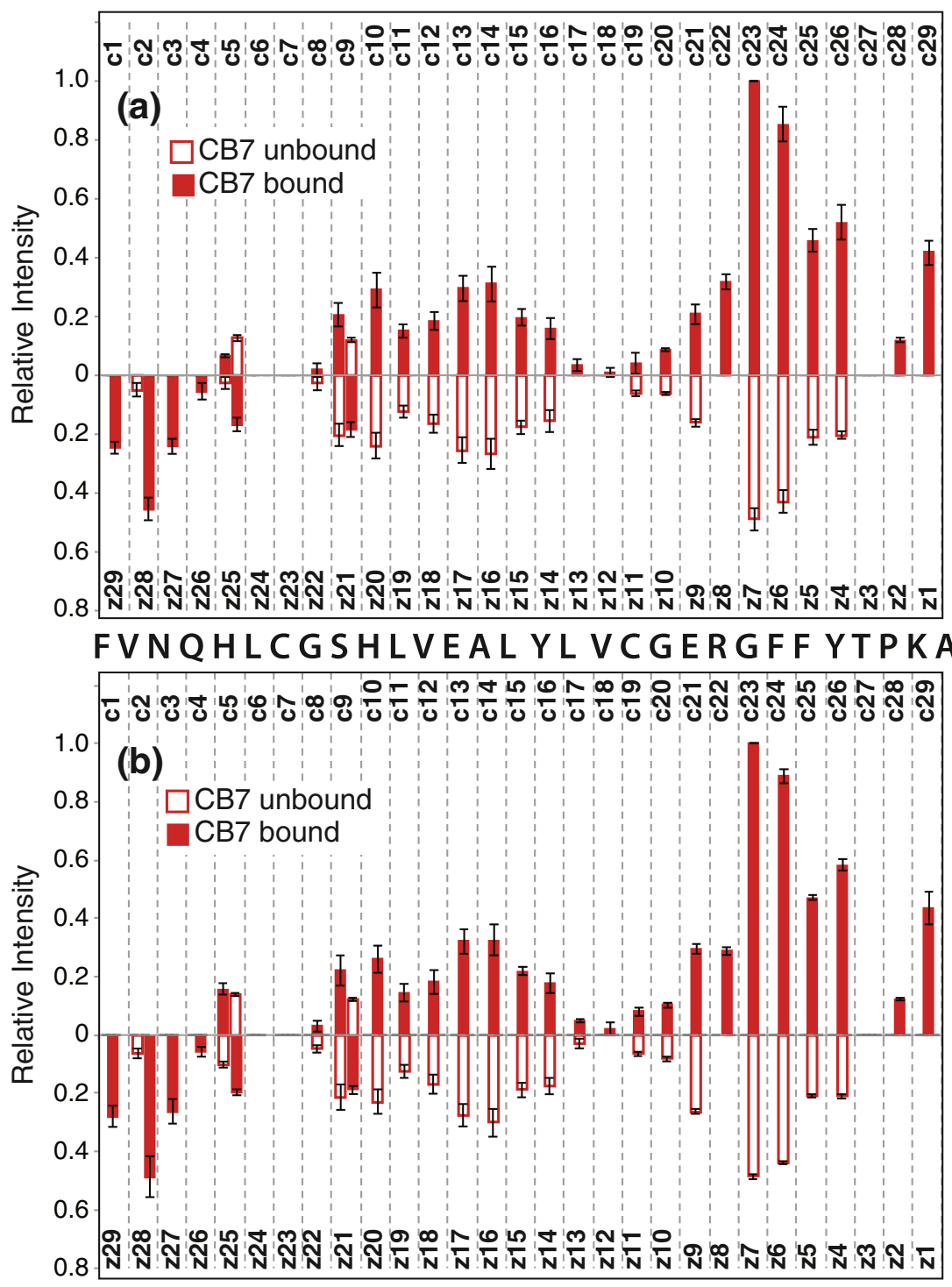

Figure 5. $E C D$ cleavage site frequencies for $\left[\mathrm{B}_{\text {red }}+\mathrm{CB} 7+5 \mathrm{H}\right]^{5+}$ at collision voltage (a) $0 \mathrm{~V}$ and (b) $-6 \mathrm{~V}$. Data are derived from the average of five trials and are normalized to the intensity of the most intense fragment, c24. Each error bar represents \pm 1 standard deviation. Bars above the ordinate represent $\mathrm{c}$-ion intensities and bars above the ordinate represent z-ion intensities. Filled red bars represent holo (CB7 bound) fragments, while unfilled bars represent the intensity of apo (CB7 unbound) fragments

complementary to the observed $2+$ holo forms of $\mathrm{c} 5$ and $\mathrm{c} 9$ and thus consistent with Phe1 binding, exist only in the $2+$ charge state, whereas holo z25 and z21 (complementary to the unexpected $1+$ apo c5 and c9 fragments) exist as both $2+$ and $3+$. These results indicate a strong correlation between the position of the CB7 ligand and the charge site locations. In particular, it appears that retention of the CB7 ligand by the c-type fragment ions is correlated to the presence of charges on both the N-terminus and His5, resulting in a doubly-charged holo fragment (i.e. holo c5 and c9). In contrast, when only one of these two sites is charged (either the N-terminus or His5), the result is a singly-charged apo fragment (i.e., apo c5 and c9), indicating loss of CB7 from the initial solution-phase binding site.
Based on the results of Figure 6, we propose that the neutralization of the charge at the $\mathrm{N}$-terminus by the capture of an electron, could result in the migration of the CB7 ligand to (or its preferential retention by) a different, positively charged site, likely the His10. Further insight into this migration process could be provided by ECD experiments on the $4+$ charge state of the complex, in which presumably only one of His5 or His 10 would be protonated. Unfortunately, examination of the $4+$ charge state of the complex is hampered by overlap of the $4+$ charge state distribution of the CB7 complex with the $3+$ charge state distribution of $\mathrm{B}_{\text {red }}$ bound to one sodium. Attempts to isolate the $3+$ charge state of $\mathrm{B}_{\text {red }}$ bound to $\mathrm{CB} 7$ in sufficient quantity for ECD analysis were also unsuccessful. 


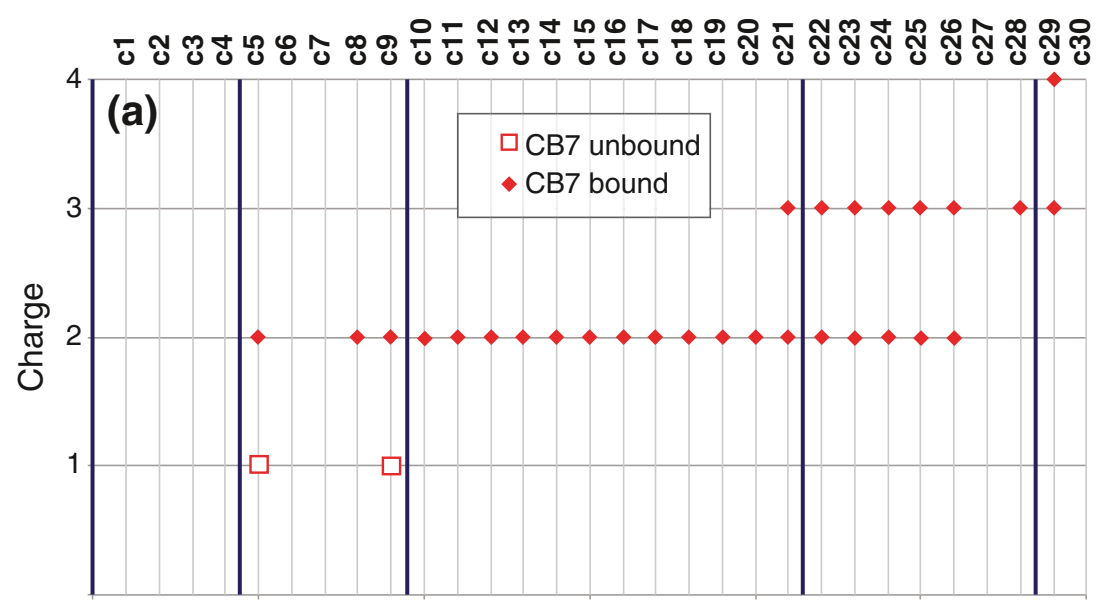

F V NQHLCGSHLVEA L Y LVCGERGFF Y T P K A

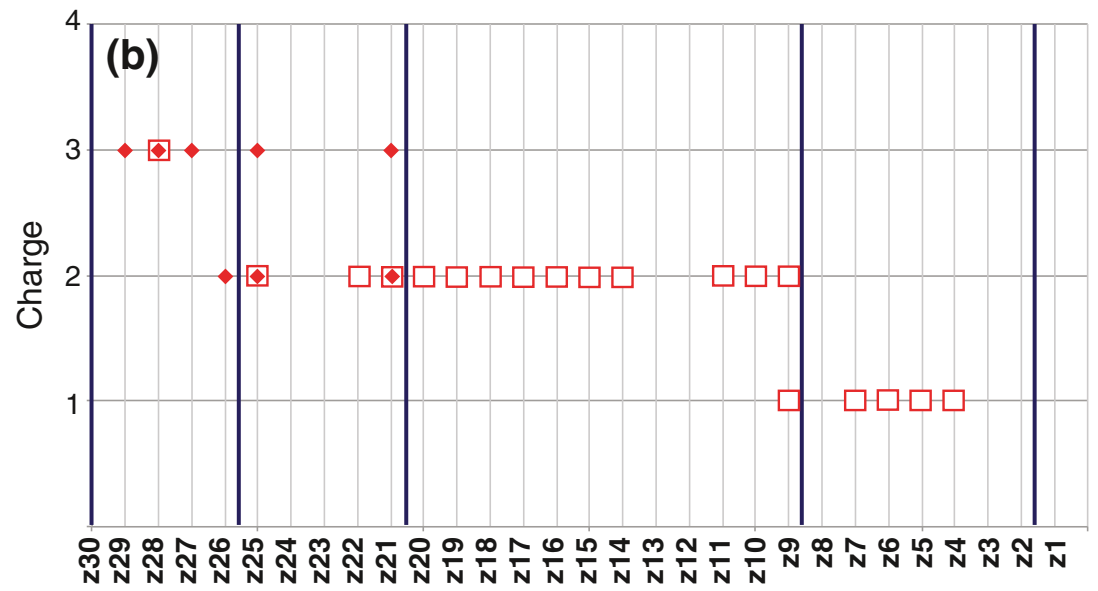

Figure 6. Charge state of the observed (a) $c$-ions and (b) z-ions from ECD of $\left[\mathrm{B}_{\text {red }}+\mathrm{CB} 7+5 \mathrm{H}\right]^{5+}$. The five vertical blue lines indicate the locations of the five most basic sites on the peptide. Red filled diamonds represent holo fragments while red open squares indicate apo fragments

We note that interaction between $\mathrm{CB} 7$ and a residue between His5 and His10, as indicated by the gas-phase ECD results, is not evident in the X-ray crystal structure, which shows the $\mathrm{N}$-terminal region of the B-chain extending away from the rest of the protein to accommodate CB7 binding (Figure 1c) [29]. In this structure, only the first few residues $\left(\mathrm{H}_{2} \mathrm{~N}-\mathrm{Phe}-\right.$ Val-Asn-) have any significant interaction with the CB7 host molecule. However, the X-ray structure may only represent a snapshot of the complex that crystallizes well. NMR results indicate that the first eight residues of the B-chain are rather flexible [35]. Unfortunately, no NMR data of the complex is available to explore the (range of) solution conformation(s) adopted. It seems probable that the $\mathrm{N}$ terminal region of gaseous insulin folds back to accommodate an interaction between CB7 and residues further down the B-chain, in addition to its interaction with PheB1. In this picture, insulin adopts a conformation significantly more compact than the extended one present in the crystal structure.

In an attempt to investigate the effects of gas-phase conditions on fragmentation products, ECD experiments were repeated with a more negative collision voltage (CV) ( $-6 \mathrm{~V}$ compared with $0 \mathrm{~V}$ used in all other experiments), which is the bias voltage used to accelerate ions as they enter the accumulation hexapole (h2), prior to ECD analysis. Increasing the collision energy has been shown to induce conformational unfolding of proteins and protein-ligand complexes in the gas phase $[39,40]$. Thus, by increasing the collision energy, we hoped to produce a more extended structure and to lower the degree of CB7 migration. Increasing the collision energy may also increase ECD efficiency as a result of increased internal energy. Mild collisional activation $(\mathrm{CV}=-6)$ prior to $\mathrm{ECD}$ results in largely similar fragment ion intensities to the $\mathrm{CV}=0$ data (Figure 5b). One noticeable difference between the two data sets is the relative intensities of the fragments resulting from the dissociation of the $\mathrm{c} 5 / \mathrm{z} 25$ bond. With $\mathrm{CV}=0$, the intensity of the apo-c5 fragment is greater than the holo form, indicating that the preferred fragmentation pathway retains CB7 on the C-terminal side of His5. Similarly, the complementary z 25 fragment is predominantly present in the holo form, again consistent with localization of the CB7 on 
the C-terminal side of His5. Conversely, at the higher collision energy, a statistically significant increase is apparent in the intensity of the holo-c5 fragment relative to the apo-c5 fragment, while the intensity of the apo-z25 has increased significantly relative to the holo-z25 fragment. This result highlights the effect of gas-phase conditions on the observed fragment ions. However, we note that increasing the collision energy has no significant effect on the relative intensities of the apo and holo c9/z21 or z28 fragments. This may be due to the presence of multiple conformations of the complex, some of which have CB7 bound or close to His5 and others with $\mathrm{CB} 7$ bound or close to His10. Alternatively, it may be that CB7 interacts with both the His5 and His 10 residues and that mild collisional activation disrupts the interaction of $\mathrm{CB} 7$ and the His5 residue but maintains the interaction with the His10 residue.

Finally, we note that CB7 binds tightly to insulin in the gas phase. Sustained off-resonance irradiation (SORI) CAD of $[\mathrm{Ins}+\mathrm{CB} 7+5 \mathrm{H}]^{5+}$ proceeded without disruption of the noncovalent complex (see supplementary information). The inferred tight-binding mirrors the host-guest chemistry observed between gaseous peptides and a protein with the smaller cucurbit[6]uril, which interacts favorably with the protonated lysine side chain $[41,42]$. The majority of SORI$\mathrm{CAD}$ fragments from $[\mathrm{Ins}+\mathrm{CB} 7+5 \mathrm{H}]^{5+}$ can be assigned to the loss of small neutrals $\left(\mathrm{H}_{2} \mathrm{O}\right.$ and $\left.\mathrm{NH}_{3}\right)$ from $\mathrm{b}$ and $\mathrm{y}$ fragment ions. Fragmentation was only observed on the Cterminal end of the A- and B-chains; $\mathrm{b}$ type fragments all retained the $\mathrm{CB} 7$, while the y type fragments did not. Unfortunately, no fragments were observed towards the $\mathrm{N}$ terminal side of the B-chain, thus SORI-CAD of the intact complex was not adequate for further localizing the binding site.

SORI-CAD of the reduced B-chain produced several Nterminal fragments (see supplementary information). These include the complementary apo b3/holo y 27 and apo b4/holo y26 fragment ion pairs, the observation of which suggest that $\mathrm{CB} 7$ is located not on the N-terminus, but further down the B-chain. The presence of holo b12-27 fragment ions further suggests that the CB7 is located (or at least upon low energy SORI-CAD is retained) between residues His5 and Val12 of the B-chain. The SORI-CAD results on the reduced insulin B-chain are thus consistent with ECD results, and favor the idea that $\mathrm{CB} 7$ migrates from its primary solutionphase interaction with the N-terminal Phe to interact most strongly with other residues, further down the B-chain, in the gas phase.

\section{Conclusions}

Here we present a cautionary tale of the use of ECD for ligand binding site determination. MS results using nanoESI and competitive binding experiments support the conclusion that the N-terminal Phe residue is essential for solution binding of CB7 to insulin, as previously indicated by isothermal titration calorimetry, fluorescence spectrosco- py, and X-ray crystallography data [29]. However, ESIECD-MS analysis suggests that migration of the ligand occurs in the gas phase, presenting a case where ECD fails to provide information reflecting the accepted solution-phase binding site. We propose that the N-terminal region of the Bchain folds back onto the surface of the insulin upon desolvation, allowing for new interactions of CB7 with charged residues on the surface of the protein. Moreover, we propose that the apparent ligand migration may be enhanced by the charge neutralization that results from the ECD process itself because of the importance of ion-dipole interactions in CB7 binding. However, the apparent migration may be simply a result of desolvation and/or the additional charges imparted by the ESI process.

Several studies have reported the use of ESI for transferring noncovalent complexes from solution into the gas phase for interrogation by ECD [22-27]. However, structural analysis using gas-phase dissociation techniques such as ECD can only provide accurate information about the solution binding sites if the gas-phase structure does not undergo significant structural changes. Additionally, the presence of multiple charges on the surface of the gas-phase structure can introduce new interactions, favorable or disfavorable, between the protein and the ligand. These concepts should always be taken into account when considering the application of MS for probing solutionphase structures. The results presented here caution against the use of ECD-MS as a stand-alone method for probing solution-phase binding sites.

\section{Acknowledgments}

The authors gratefully acknowledge support for this work provided by the Canada Foundation for Innovation, the Natural Sciences and Engineering Research Council of Canada, the Canada Research Chairs program, and the Province of Ontario.

\section{References}

1. Loo, J.: Studying noncovalent protein complexes by electrospray ionization mass spectrometry. Mass Spectrom. Rev. 16, 1-23 (1997)

2. Heck, A.J.R., van den Heuvel, R.H.H.: Investigation of intact protein complexes by mass spectrometry. Mass Spectrom. Rev. 23, 368-389 (2004)

3. Wyttenbach, T., Bowers, M.T.: Intermolecular interactions in biomolecular systems examined by mass spectrometry. Annu. Rev. Phys. Chem. 58, 511-533 (2007)

4. Breuker, K.: The study of protein-ligand interactions by mass spectrometry-a personal view. Int. J. Mass Spectrom. 239, 33-41 (2004)

5. Borch, J., Jørgensen, T.J., Roepstorff, P.: Mass spectrometric analysis of protein interactions. Curr. Opin. Chem. Biol. 9, 509-516 (2005)

6. Sharon, M., Robinson, C.V.: Peeling back the layers of complexity. Curr. Opin. Struct. Biol. 21, 619-621 (2011)

7. Hernández, H., Robinson, C.V.: Determining the stoichiometry and interactions of macromolecular assemblies from mass spectrometry. Nat. Protoc. 2, 715-726 (2007)

8. Zhou, M., Robinson, C.V.: When proteomics meets structural biology. Trends Biochem. Sci. 35, 522-529 (2010)

9. Heck, A.J.R.: Native mass spectrometry: a bridge between interactomics and structural biology. Nat. Methods 5, 927-933 (2008) 
10. Kruppa, G.H., Schoeniger, J., Young, M.M.: A top down approach to protein structural studies using chemical cross-linking and Fourier transform mass spectrometry. Rapid Commun. Mass Spectrom. 17, 155-162 (2003)

11. Ihling, C., Schmidt, A., Kalkhof, S., Schulz, D.M., Stingl, C., Mechtler, K., Hack, M., Beck-Sickinger, A.G., Cooper, D.M.F., Sinz, A.: Isotopelabeled cross-linkers and Fourier transform ion cyclotron resonance mass spectrometry for structural analysis of a protein/peptide complex. J. Am. Soc. Mass Spectrom. 17, 1100-1113 (2006)

12. Guan, J.-Q., Chance, M.R.: Structural proteomics of macromolecular assemblies using oxidative footprinting and mass spectrometry. Trends Biochem. Sci. 30, 583-592 (2005)

13. Gau, B., Garai, K., Frieden, C., Gross, M.L.: Mass spectrometry-based protein footprinting characterizes the structures of oligomeric apolipoprotein E2, E3, and E4. Biochemistry 50, 8117-8126 (2011)

14. Zubarev, R.A., Kelleher, N.L., McLafferty, F.W.: Electron capture dissociation of multiply charged protein cations. A nonergodic process. J. Am. Chem. Soc. 120, 3265-3266 (1998)

15. Syka, J.E.P., Coon, J.J., Schroeder, M.J., Shabanowitz, J., Hunt, D.F.: Peptide and protein sequence analysis by electron transfer dissociation mass spectrometry. Proc. Natl. Acad. Sci. U.S.A. 101, 9528-9533 (2004)

16. Meng, F., Forbes, A.J., Miller, L.M., Kelleher, N.L.: Detection and localization of protein modifications by high resolution tandem mass spectrometry. Mass Spectrom. Rev. 24, 126-134 (2005)

17. Siuti, N., Kelleher, N.L.: Decoding protein modifications using topdown mass spectrometry. Nat. Methods 4, 817-821 (2007)

18. McLafferty, F.W., Fridriksson, E.K., Horn, D.M., Lewis, M.A., Zubarev, R.A.: Biomolecule mass spectrometry. Science 284, 12891290 (1999)

19. Sze, S.K., Ge, Y., Oh, H., McLafferty, F.W.: Top-down mass spectrometry of a $29-\mathrm{kDa}$ protein for characterization of any posttranslational modification to within one residue. Proc. Natl. Acad. Sci. U.S.A. 99, 1774-1779 (2002)

20. Zubarev, R.A., Horn, D.M., Fridriksson, E.K., Kelleher, N.L., Kruger, N.A., Lewis, M.A., Carpenter, B.K., McLafferty, F.W.: Electron capture dissociation for structural characterization of multiply charged protein cations. Anal. Chem. 72, 563-573 (2000)

21. Haselmann, K.F., Jørgensen, T.J.D., Budnik, B.A., Jensen, F., Zubarev, R.A.: Electron capture dissociation of weakly bound polypeptide polycationic complexes. Rapid Commun. Mass Spectrom. 16, 22602265 (2002)

22. Xie, Y., Zhang, J., Yin, S., Loo, J.A.: Top-down ESI-ECD-FT-ICR mass spectrometry localizes noncovalent protein-ligand binding sites. $J$. Am. Chem. Soc. 128, 14432-14433 (2006)

23. Yin, S., Loo, J.A.: Elucidating the site of protein-ATP binding by topdown mass spectrometry. J. Am. Soc. Mass Spectrom. 21, 899-907 (2010)

24. Yin, S., Loo, J.A.: Top-down mass spectrometry of supercharged native protein-ligand complexes. Int. J. Mass Spectrom. 300, 118-122 (2011)

25. Zhang, H., Cui, W., Wen, J., Blankenship, R.E., Gross, M.L.: Native electrospray and electron-capture dissociation FTICR mass spectrometry for top-down studies of protein assemblies. Anal. Chem. 83, 55985606 (2011)

26. Clarke, D.J., Murray, E., Hupp, T., Mackay, C.L., Langridge-Smith, P.R.R.: Mapping a noncovalent protein-peptide interface by top-down FTICR mass spectrometry using electron capture dissociation. $J$. Am. Soc. Mass Spectrom. 22, 1432-1440 (2011)

27. Sinha, S., Lopes, D.H.J., Du, Z., Pang, E.S., Shanmugam, A., Lomakin, A., Talbiersky, P., Tennstaedt, A., McDaniel, K., Bakshi, R., Kuo,
P.-Y., Ehrmann, M., Benedek, G.B., Loo, J.A., Klärner, F.-G., Schrader, T., Wang, C., Bitan, G.: Lysine-specific molecular tweezers are broad-spectrum inhibitors of assembly and toxicity of amyloid proteins. J. Am. Chem. Soc. 133, 16958-16969 (2011)

28. Enyenihi, A.A., Yang, H., Ytterberg, A.J., Lyutvinskiy, Y., Zubarev, R.A.: Heme binding in gas-phase holo-myoglobin cations: distal becomes proximal? J. Am. Soc. Mass Spectrom. 22, 1763-1770 (2011)

29. Chinai, J.M., Taylor, A.B., Ryno, L.M., Hargreaves, N.D., Morris, C.A., Hart, P.J., Urbach, A.R.: Molecular recognition of insulin by a synthetic receptor. J. Am. Chem. Soc. 133, 8810-8813 (2011)

30. Rekharsky, M.V., Yamamura, H., Ko, Y.H., Selvapalam, N., Kim, K., Inoue, Y.: Sequence recognition and self-sorting of a dipeptide by cucurbituril and cucurbituril. Chem. Commun. 2236 (2008)

31. Zamdborg, L., LeDuc, R.D., Glowacz, K.J., Kim, Y.-B., Viswanathan, V., Spaulding, I.T., Early, B.P., Bluhm, E.J., Babai, S., Kelleher, N.L.: ProSight PTM 2.0: improved protein identification and characterization for top down mass spectrometry. Nucleic Acids Res. 35, W701-W706 (2007)

32. Mock, W.L., Shih, N.-Y.: Structure and selectivity in host-guest complexes of cucurbituril. J. Org. Chem. 51, 4440-4446 (1986)

33. Mock, W.L., Shih, N.-Y.: Organic ligand-receptor interactions between cucurbituril and alkylammonium ions. J. Am. Chem. Soc. 110, 47064710 (1988)

34. Shneine, J., Voswinkel, M., Federwisch, M., Wollmer, A.: Enhancing the $\mathrm{T}->\mathrm{R}$ transition of insulin by helix-promoting sequence modifications at the N-terminal B-chain. Biol. Chem. 381, 127-133 (2000)

35. Hua, Q., Weiss, M.A.: Comparative 2D NMR studies of human insulin and despentapeptide insulin: sequential resonance assignment and implications for protein dynamics and receptor recognition. Biochemistry 30, 5505-5515 (1991)

36. Zubarev, R.A., Kruger, N.A., Fridriksson, E.K., Lewis, M.A., Horn, D.M., Carpenter, B.K., McLafferty, F.W.: Electron capture dissociation of gaseous multiply-charged proteins is favored at disulfide bonds and other sites of high hydrogen atom affinity. J. Am. Chem. Soc. 121, 2857-2862 (1999)

37. Hennig, A., Bakirci, H., Nau, W.M.: Label-free continuous enzyme assays with macrocycle-fluorescent dye complexes. Nat. Methods 4, 629-632 (2007)

38. Bailey, D.M., Hennig, A., Uzunova, V.D., Nau, W.M.: Supramolecular tandem enzyme assays for multiparameter sensor arrays and enantiomeric excess determination of amino acids. Chem. Eur. J. 14, 60696077 (2008)

39. Hopper, J.T.S., Oldham, N.J.: Collision induced unfolding of protein ions in the gas phase studied by ion mobility-mass spectrometry: the effect of ligand binding on conformational stability. J. Am. Soc. Mass Spectrom. 20, 1851-1858 (2009)

40. Skinner, O.S., McLafferty, F.W., Breuker, K.: How ubiquitin unfolds after transfer into the gas phase. J. Am. Soc. Mass Spectrom. 23, 10111014 (2012)

41. Zhang, H., Grabenauer, M., Bowers, M.T., Dearden, D.V.: Supramolecular Modification of Ion Chemistry: Modulation of Peptide Charge State and Dissociation Behavior through Complexation with cucurbituril $(\mathrm{n}=5,6)$ or alpha-Cyclodextrin. J. Phys. Chem. A 113, 1508-1517 (2009)

42. Heo, S.W., Choi, T.S., Park, K.M., Ko, Y.H., Kim, S.B., Kim, K., Kim, H.I.: Host-Guest Chemistry in the Gas Phase: Selected Fragmentations of CB[6]-Peptide Complexes at Lysine Residues and Its Utility to Probe the Structures of Small Proteins. Anal. Chem. 83, 7916-7923 (2011)

43. Schrödinger, L.L.C.: The PyMOL Molecular Graphics System, version $1.3 \mathrm{r} 1,(2010)$ 